\title{
Plasma spermidine concentrations as early indication of response to therapy in human myeloma
}

\author{
OA VAN DOBBENBURGH, B HOUWEN*, H JURJENS $\dagger$, J MARRINK $\ddagger$, MR HALIE, HO \\ NIEWEG
}

From the Departments of Haematology, Nuclear Medicine $\dagger$ and Laboratory of Immunochemistry $\ddagger$, University Hospital, Groningen, The Netherlands.

SUMMARY Eighteen patients with melphalan refractory myeloma were treated with vindesine and $\dot{\omega}_{\omega}$ prednisone. Plasma spermidine concentrations were measured by radioimmunoassay before and -7 after a single vindesine injection. Seven patients showed a significant rise of plasma spermidine after vindesine and five of these showed a clinical response on further evaluation. Of the 11 patients who did not show raised spermidine concentrations, 10 did not respond to the therapy. The correlation between clinical response/rise of spermidine and between non-response/no rise of spermidine was statistically significant $(\mathrm{p}<0 \cdot 05)$. Pretreatment spermidine concentrations did $\stackrel{\sum}{\gtrless}$ not distinguish those who responded to treatment nor did they differ in patients and controls.

Measurement of polyamines, mainly spermidine and putrescine, has been shown to be useful in experimental research especially concerning tumour growth and response to treatment.

Cell death is reflected in an increase of the spermidine concentration in biological fluids-for example, urine, serum, or cerebrospinal fluidpossibly because spermidine is released by the dying cell. ${ }^{2}$ In recent years a considerable amount of work has been done to establish practical clinical utility for polyamine measurements. In 1975 Russell et al showed that an increase of spermidine excretion in urine after cytostatic treatment correlated with clinical response $^{3}$ in solid and haematological tumours. These results were extended and confirmed. ${ }^{4}$ Since 1977 , however, the only clinical application for measurement of polyamines has been monitoring recurrence of medulloblastoma by measuring putrescine concentrations in CSF. ${ }^{1}$ One of the possible reasons for a decline in interest ${ }^{1}$ is that most polyamine determinations were done on 24-hour urine. Samples are cumbersome to obtain and their collection may be incomplete. Recently, a radioimmunoassay for spermidine measurement in plasma became available, and prompted by the studies of Russell et al $^{34}$ we investigated the possibility of drug sensitivity testing in vivo.

\footnotetext{
*Present address: Department of Haematology, Foothills Hospital, Calgary, Alberta, Canada.
}

Accepted for publication 27 January 1983
In this paper we present the results of plasma spermidine measurements before and after an injection of vindesine in patients with melphalanresistant multiple myeloma. The correlation of the spermidine concentration with the clinical response $\stackrel{\mathbb{Q}}{\mathbb{Q}}$ is investigated.

\section{Patients, material and methods}

Spermidine concentrations were measured by a radio immunoassay. ${ }^{5}$ Antibodies to spermidine were? raised in rabbits by immunisation with spermidine $\frac{9}{3}$ covalently bound to bovine thyroglobulin. Plasma spermidine concentrations were estimated by using $O$ a double-antibody technique. Only free spermidine

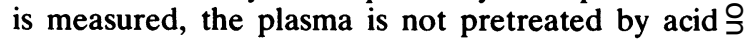
hydrolysis. Cross reaction with spermine or putres- $D$ cine is less than $1 \%$. Spermidine concentrations are expressed in $\mathrm{nmol} / \mathrm{l}$. Venous blood samples were $N$ taken in heparinised vacuum glass tubes. Directly after venepuncture the blood was centrifuged $(3000 \stackrel{\sim}{\circ}$ $\mathrm{rpm}$ for $10 \mathrm{~min}$ ) and plasma was frozen and stored at $\mathrm{N}$ $-20^{\circ}$. Haemolytic samples were discarded. The spermidine assay took place within 14 days aftero sampling.

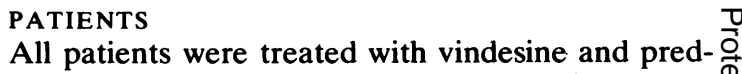
nisone for multiple myeloma resistant to melphalan/prednisone treatment. The preliminary $\stackrel{\square}{\square}$ results of this have been published. 6 Eighteen patients participated in the present study. 
Vindesine was given as an intravenous bolus injection $\left(2 \mathrm{mg} / \mathrm{m}^{2}\right)$ at weekly intervals for three consecutive weeks, followed by a three week therapy-free interval. After each vindesine injection prednisone was given orally in daily doses of $100 \mathrm{mg}$ for five days. Evaluation of response took place after three months of treatment, response was measured according to the Southwest Oncology Group (SWOG)-criteria ${ }^{7}$ which differentiate between "responders," "improved patients" and "nonresponders". In this paper the improved patients as well as the responders are called "responders." All patients were classified according to Durie ${ }^{8}$ as stage III disease (high tumour load).

\section{PROTOCOL}

Patients were admitted to hospital for their first vindesine injection. This injection was not followed by prednisone because of the probable stimulating affect of prednisone on polyamine metabolism. ${ }^{9}$ The baseline plasma sample was taken at $0800 \mathrm{~h}$ and vindesine was administered immediately afterwards. Further samples were taken at 2, 4, 6 and $8 \mathrm{~h}$ after the vindesine injection and daily at $0800 \mathrm{~h}$ for the next five or six days. Patients were free from bacterial infections. As controls, samples were taken from 12 healthy volunteers at $0800,1100,1300$ and 1600 h.

\section{STATISTICS}

For correlation of response and spermidine concentration the $2 \times 2$ contingency tables were used.

\section{Results}

Pretreatment values of the patients are shown in Fig. 1 (range $50-920 \mathrm{nmol} / \mathrm{l}$, mean $290 \mathrm{nmol} / \mathrm{l}$ ) and they are compared with the $0800 \mathrm{~h}$ values of the 12 controls (range $90-550$, mean $270.5 \mathrm{nmol} / \mathrm{l}$ ).

Although two of the patients had very high pretreatment values it seems that these initial values do not discriminate between patients and controls, nor do they discriminate between responders to treatment and non-responders (open $v$ closed circles, Fig. 1). Spermidine concentrations of the $\mathbf{1 2}$ healthy volunteers over the day are shown in Fig. 2. Values are expressed as a percentage of the $0800 \mathrm{~h}$ sample.

A diurnal fluctuation of $\pm 50 \%$ was observed. The spermidine values, after the vindesine injection expressed as a percentage of the pretreatment value, are shown in Fig. 3. Because of the diurnal fluctuation observed in the controls we considered that a change in spermidine values of $\geqslant 100 \%$ in the patients after treatment was significant. The patients were classified according to (a) response versus non-response to treatment and (b) significant versus
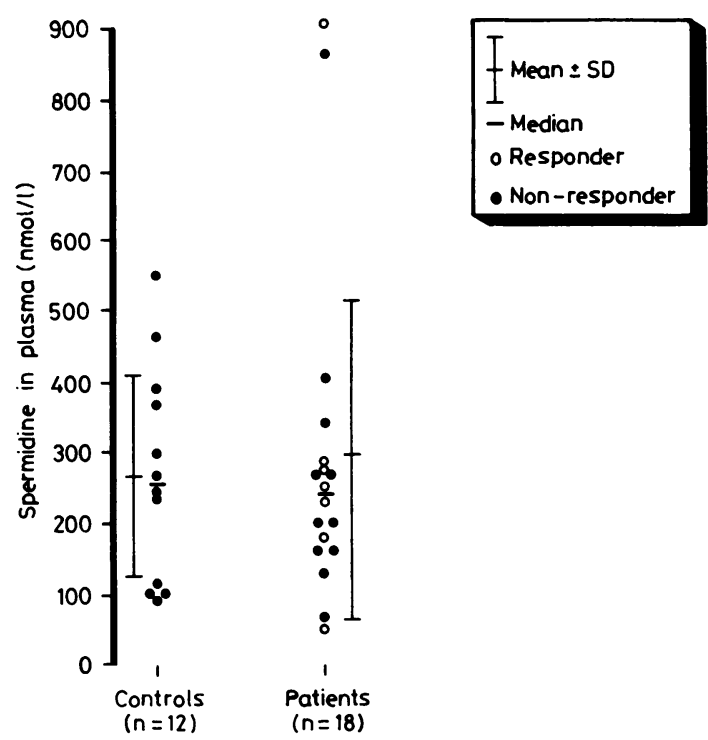

Fig. 1 Baseline values of controls (left) and patients (right). Responders are indicated by open circles.

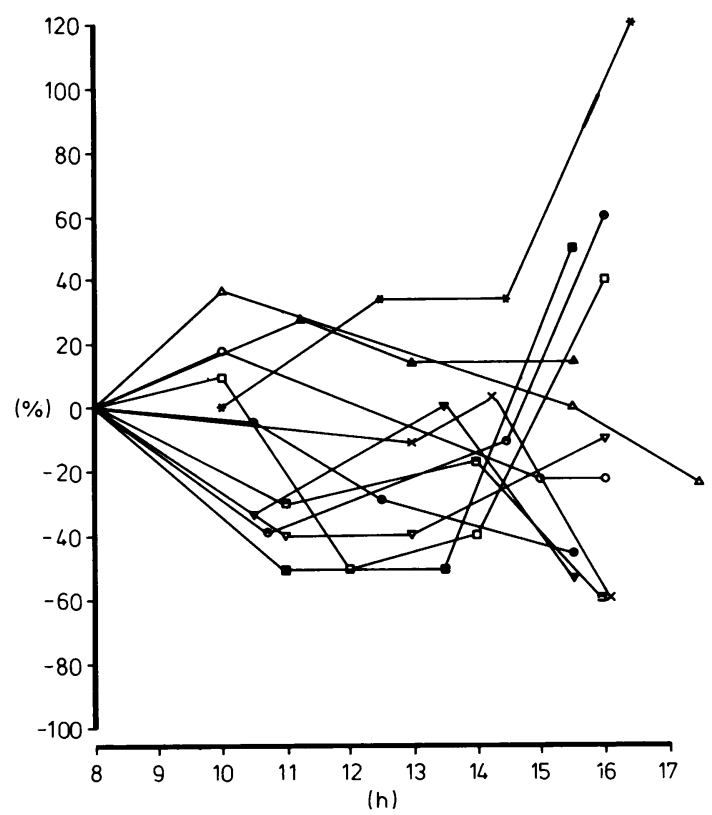

Fig. 2 Spermidine values (expressed as a percentage of the $0800 \mathrm{~h}$ values) at approximately $1100 \mathrm{~h}, 1300 \mathrm{~h}$ and $1600 \mathrm{~h}$ of 12 controls. One curve (*) starts at $1000 \mathrm{~h}$.

the non-significant rise of spermidine concentration.

Five of the six responders showed a spermidine rise of $>100 \%$ while ten of the twelve nonresponders did not show a significant rise (Table). 


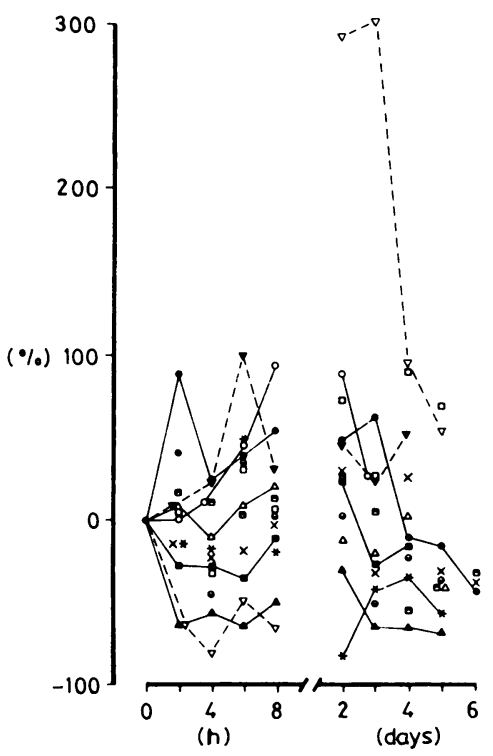

Table Correlation between response/non-response and spermidine rise/non-spermidine rise $(p<0.05)$

\begin{tabular}{llcr}
\hline & \multicolumn{2}{l}{ Significant spermidine rise } & \\
\cline { 2 - 3 } & + & - & Sensitivity $83 \%^{*}$ \\
Response & 5 & 1 & Specificity $83 \% \dagger$ \\
Non-response & 2 & 10 & \\
Total & 7 & 11 & \\
& PV pos $71 \% \ddagger P V$ neg $91 \%$ 8 \\
\hline
\end{tabular}

*Sensitivity: true positive ratio.

†Specificity: true negative ratio.

$\ddagger P V$ pos: predictive value of a positive test result.

$\$ P V$ neg: predictive value of a negative test result.

The correlation between response/non-response and significant/non-significant spermidine rise was statistically significant $(p<0.05)$.

From the same figures a decision matrix was calculated (Table). Sensitivity and specificity were both $83 \%$. The predictive value of a significant spermidine rise was $71 \%$; the predictive value of absence of a spermidine rise was $91 \%$.

\section{Discussion}

Recently a radioimmunoassay for plasma spermidine concentration became available to us. In patients with melphalan-resistant multiple myeloma who were treated with vindesine and prednisone we studied plasma spermidine concentrations after vindesine administration. Although all patients had
Fig. 3 Spermidine values (expressed as a percentage of the baseline values) of responding (right) and non-responding (left, not all curves drawn) patients. Dashed lines indicate two nonresponders with a spermidine rise and one (right) responder without a spermidine rise.

stage III disease, only two patients showed extremely high pretreatment values, probably indicating increased spontaneous cell death. This could indicate different tumour kinetics in individual $\stackrel{ }{\circ}$ patients and is in accordance with the view of Durie $^{10}$ who demonstrated different tumour doubl- $\bar{\Phi}$ ing times within one group of patients with the same stage of disease. The spermidine concentrations of the responding patients showed a rise within $24 h_{J}$ indicating cell death quite soon after the vindesine? administration, which agrees with in vitro experiments. ${ }^{11}$ It also correlates with our clinical observa -8 tion of rapidly decreasing skeletal pain in severalo patients. Both observations suggest that plasma. spermidine concentrations can be used for researchô on drug and tumour kinetics.

Pretreatment spermidine concentrations did not윽 distinguish patients from controls, nor between $>$ those who responded to treatment or those who did을. not. A statistically significant correlation was shown between a significant rise of the plasma spermidine. value (after drug administration) and a clinical ${ }_{\circ}^{N}$ response. With the spermidine values obtained in this particular protocol, response and especiallyo non-response could be predicted with reasonableo accuracy (decision matrix, see Results). If this (or similar) protocol could be used to predict response? or non-response to other cytostatic drugs, the possi- 0 bility arises to test tumour drug sensitivity in vivo in웅 the individual patient. In analogy to tumour drug sensitivity testing in vitro (tumour stem cell assay) $\mathbb{Q}$ treatment could then be selected on an individualo basis. 
This could be especially useful in malignancies in which response or non-response to treatment is not always readily apparent for example, multiple myeloma, where the prolonged half life of the M-protein may delay response evaluation. The radioimmunoassay for spermidine is a rapid method, so drug sensitivity testing for one or more drugs should enable the physician to select proper treatment shortly after his decision to treat the patient.

Since the present study is limited to a small group of patients, in which only one drug is tested for one disease, further studies are needed to confirm the usefulness of plasma spermidine measurement in tumour drug sensitivity testing.

We are grateful to $\mathrm{Mr} \mathbf{J}$ Bijzet for technical assistance in development of the spermidine radioimmunoassay.

\section{References}

${ }^{1}$ Marton LJ, Seidenfeld J. Approaches to the study of polyamines as cancer markers. In: Morris DR, Marton LJ, eds. Polyamines in biology and medicine (Vol. 8 in The biochemistry of disease. M Dekker, 1981, 337-348.
${ }^{2}$ Milano G, Schneider M, Cassuto JP, et al. Polyamines: comments on clinical utility in cancer. A review. Tumor Diagnostik 1980; 3:121-5.

${ }^{3}$ Russell DH, Durie BGM, Salmon SE. Polyamines as predictors of success and failure in cancer chemotherapy. Lancet 1975;ï:797-9.

4 Durie BGM, Salmon SE, Russell DH. Polyamines as markers of response and disease activity in cancer chemotherapy. Cancer Res 1977;37:214-21.

5 Jurjens $\mathbf{H}$, Bijzet J, Woldring MG. Radioimmuno assay of spermidine in extracted plasma. Clin Chim Acta 1983; (in press).

${ }^{6}$ Houwen B, Ockhuizen Th, Marrink J, Nieweg HO. Vindesine therapy in melphalan-resistant multiple myeloma. Eur $J$ Cancer 1981;17:227-32.

' Alexanian R, Bonnet J, Gehan E, et al. Combination chemotherapy for multiple myeloma. Cancer 1972;30:382-9.

${ }^{8}$ Durie BGM, Salmon SE. A clinical staging system for multiple myeloma. Cancer 1975;36:842-54.

9 Jänne J, Pösö H, Raina A. Polyamines in rapid growth and cancer. Biochem Biophys Acta 1978;473:241-93.

${ }^{10}$ Durie BGM. Staging and kinetics of multiple myeloma. Clinics in hematology. Philadelphia: W B Saunders, 1982:3-18.

"Hill BT, Whelan RDH. Comparitive effects of vincristine and vindesine on cell cycle kinetics in vitro. Cancer Treatment Rev 1980; 7(suppl):5-15.

Requests for reprints to: $\mathrm{Dr} O \mathrm{O}$ A van Dobbenburgh, University Hospital, Oostersingel 59,9713 EZ Groningen, The Netherlands. 